This item was submitted to Loughborough's Research Repository by the author.

Items in Figshare are protected by copyright, with all rights reserved, unless otherwise indicated.

\title{
Application of wireless inertial measurement units and EMG sensors for studying deglutition - preliminary results
}

PLEASE CITE THE PUBLISHED VERSION

http://dx.doi.org/10.1109/EMBC.2014.6944842

PUBLISHER

(C) IEEE

VERSION

AM (Accepted Manuscript)

LICENCE

CC BY-NC-ND 4.0

\section{REPOSITORY RECORD}

Imtiaz, U., K. Yamamura, Weisheng Kong, Salvatore Sessa, Zhuohua Lin, Luca Bartolomeo, Hiroyuki Ishii, Massimiliano Zecca, Y. Yamada, and Atsuo Takanishi. 2019. "Application of Wireless Inertial Measurement Units and EMG Sensors for Studying Deglutition - Preliminary Results". figshare.

https://hdl.handle.net/2134/17597. 


\title{
Application of Wireless Inertial Measurement Units and EMG Sensors for studying Deglutition - Preliminary Results
}

\author{
U. Imtiaz, K. Yamamura, W. Kong, S. Sessa, Member, IEEE, Z. Lin, Member, IEEE, L. Bartolomeo, \\ Member, IEEE, H. Ishii, Member, IEEE, M. Zecca, Member, IEEE, Y. Yamada, and A. Takanishi, \\ Member, IEEE
}

\begin{abstract}
Different types of sensors are being used to study deglutition and mastication. These often suffer from problems related to portability, cost, reliability, comfort etc. that make it difficult to use for long term studies. An inertial measurement based sensor seems a good fit in this application; however its use has not been explored much for the specific application of deglutition research. In this paper, we present a system comprised of an IMU and EMG sensor that are integrated together as a single system. With a preliminary experiment, we determine that the system can be used for measuring the head-neck posture during swallowing in addition to other parameters during the swallowing phase. The EMG sensor may not always be a reliable source of physiological data especially for small clustered muscles like the ones responsible for swallowing. In this case, we explore the possibility of using gyroscopic data for the recognition of deglutition events.
\end{abstract}

\section{INTRODUCTION}

Swallowing is characterized as the physiological process of transportation of solids and liquids from the mouth to the stomach. It is one of the most important processes for normal human function, being directly related to dietary intake. Hence any problems or diseases related to swallowing can have debilitating consequences for the patient. Swallowing comprises of three phases: the oral, pharyngeal, and

This research has been supported by the JSPS Scientific Research-C grant [24500616] from JSPS, the JSPS Grant-in-Aid for Young Scientists (Wakate B) [25750259], the Global COE Program "Global Robot Academia", MEXT, Japan, and the Consolidated Research Institute for Advanced Science and Medical Care, Waseda University (ASMeW). It was also partially supported by a grant by STMicroelectronics, which also provided the core sensors and the microcontroller.

U. Imtiaz and W. Kong are with the Graduate School of Advanced Science and Engineering, Waseda University, Tokyo, Japan. (phone: +81-3-5369-7369; fax: $\quad+81-3-5269-9061 ; \quad$ e-mail: contact@takanishi.mech.waseda.ac.jp).

K. Yamamura is with the Division of Oral Physiology, Department of Oral Biological Sciences, Niigata University Graduate School of Medical and Dental Sciences, Niigata, Japan.

S. Sessa is with the Graduate School of Creative Science and Engineering, Waseda University, Tokyo, Japan.

Z. Lin is with the School of Mechanical and Automotive Engineering, South China University of Technology, Guangzhou, China.

L. Bartolomeo is with the Global Robot Academia, Waseda University, Tokyo, Japan.

H. Ishii is with the Waseda Research Institute for Science and Engineering, Waseda University, Tokyo, Japan.

M. Zecca is with the School of Electronic, Electrical and Systems Engineering, Loughborough University, UK

Y. Yamada is with the Oral Health Science Center, Tokyo Dental College, Tokyo, Japan.

A. Takanishi is with the Department of Modern Mechanical Engineering, Waseda University, and Humanoid Robotics Institute, Waseda University, Tokyo, Japan. esophageal phase. Of these, the oral and the pharyngeal phases are the most difficult to measure [1]. The exact mechanics of the swallowing process still remain unknown. In fact, the relative timings and the contribution of various muscles to the swallowing process are not consistent in literature [2]

No single unique solution exists to study the swallowing process especially for diagnostic as well as research purposes. No sensor provides the complete information of the entire process by itself, hence requiring a combination of multiple sensors of different types. A number of studies have been done over the years by using different types and combinations of sensors. These include using videofluoroscopy [3][4], piezoelectric sensors [5][6], electromyography [4][6][7], Electroencephalography [7], electrolaryngography [8], microphones [9] and pressure sensors [1][2][4] etc. In addition, we note that Inertial Measurement Units (IMUs) have not been used in swallowing studies except for tracking limb movements [9]. For using sensors with human subjects, especially for patients, there are several constraints that must be considered. These include comfort, safety, reliability, non-invasiveness, cost and portability. Many swallows occur unconsciously throughout the day and also during sleep [7]. Usually, the sensors used in previous studies were not portable and were either, tethered to monitoring systems by cables that make it inconvenient for long term sensing for many hours or used local storage without any means to view real-time data [5]. To better understand the role of various parts in swallowing, good data on physiological processes are necessary. Therefore, there is a need for a system that can help collect accurate empirical and quantitative data related to this process. The various sensors used should also be synchronized in order to better understand the relative timings of different processes involved.

The aim of this work is to present the development of a new system using a new combination of sensors for measuring swallowing for several hours. The aspects currently under measurement for swallowing study for our purpose are; the head movement, posture and the electromyographic activity during swallowing process. For the preliminary study, we postulated that the hyoid movement during bolus swallowing caused a small change in the relative head-neck angle depending on the head orientation. Head and neck postures are known to affect the dysphagia limit [6]. Hence, it is important to measure this parameter. This movement and the accompanying electromyographic signals were recorded with the developed system and subsequently analyzed.

Surface EMG is an electrophysiological procedure that measures skeletal muscular activity at the skin. It is relatively simple, non-invasive and has a low level of discomfort. 
However, the disadvantage of surface EMG is that the measurement of signals for swallowing may be influenced by many factors such as soft tissue and skin conditions of the neck. Furthermore, the signals themselves can be distorted due to muscle cross-talk. However, the EMG signals still provide us with the indication of the swallowing onset. In the future, we intend to combine this information with other sensor domains as well.

\section{Methodology}

\section{A. Experimental Setup}

Four healthy male subjects, including one author (WK), participated in the pilot test. For the experiment, the parameters measured were: 1 . Head angle relative to the neck, 2. Suprahyoid muscle activity.

For measuring the head movement, a miniaturized CAN-connected IMU, named WB-4R, was used. This IMU was developed in our group and primarily contains a mother board containing a 3-axis gyroscope, a 3-axis accelerometer, and a 3-axis magnetometer [10]. The mother board can be connected to either a Bluetooth module for direct wireless communication, or to a CAN transceiver board for wired connections. Two WB-4R units were used for measuring the head-neck angle. One IMU was tied to the back of the head with a Velcro band. As the head is considerably rigid, the exact placement does not matter. The second IMU was fixed over the seventh cervical vertebra, C7 [11], with medical grade tape. This is because $\mathrm{C} 7$ is very close to the relative axis of rotation between the cervical spine and the trunk [12].

An optical marker based position tracking system, the Optotrak $^{\mathrm{TM}}$ Certus Motion Capture system, was used to verify the motion of the IMUs. Each IMU was mounted in a 3D printed case with four circular depressions at the corners. Four markers were affixed on each IMU over the matching depressions. The markers were 'active-type' and strobed at 3 KHz. A single Optotrak Position sensor was placed $4 \mathrm{~m}$ behind the subject to capture the marker movement.

For monitoring the electromyographic activity during swallowing, an EMG sensor was used which was developed in our group as well [13]. This EMG sensor uses copper core Gold plated dry electrodes for sensing. The idea was to create an inexpensive biopotential signal acquisition platform that could be readily integrated with our other sensors following a similar protocol while remaining cost effective. The sensor has a fixed gain of 1500 , with a $20 \mathrm{~Hz} \sim 450 \mathrm{~Hz}$ signal band. The signal is sampled using an ARM Cortex-M1 microcontroller with a 12-bit ADC at a sampling rate of $1 \mathrm{KHz}$. These sensors were attached underneath the subject's chin, over the suprahyoid muscle using medical grade tape [14]. Before attaching the electrodes, the skin at the site was cleaned by
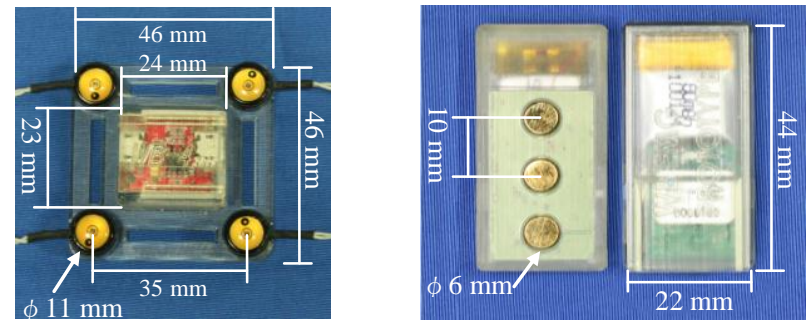

Fig. 2 A) WB-4R IMU(11.36g), Optotrak ${ }^{\mathrm{TM}}$ Markers B) WB-EMG (13.43g)
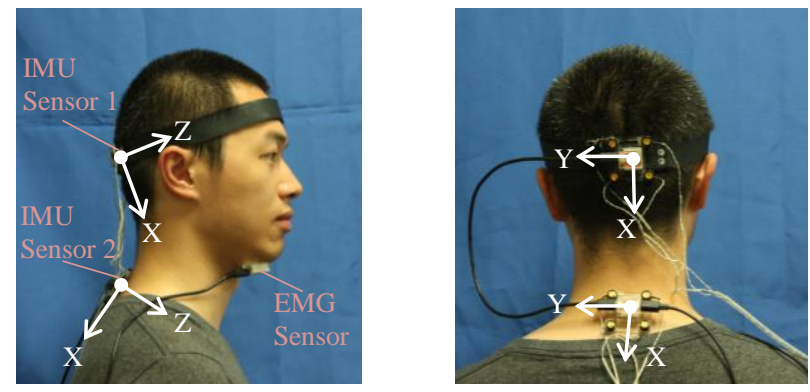

Fig. 1 Sensor placement on the body and Frame axes

gauze with alcohol. The EMG sensor was also connected to the CAN bus.

Finally, for data communication, a separate module was used, dubbed as the 'Central Board', which polls all the sensors connected to the CAN bus, packages the data and transmits to the PC wirelessly over Bluetooth. The data are recorded in the $\mathrm{PC}$ and processed.

The sensor system and the recording program as well as post processing algorithm was tested several times by mounting the setup on a tripod and moving the sensors manually about various angles, with a video recording. The angles from IMU and the Optotrak were then found to be in agreement. Details of the comparison have been published elsewhere in [15]. RMS Error of WB-4R compared to Optotrak is given in Table I.

TABLE I

RMS ERROR OF WB-4R COMPARED WITH OPTOTRAK

\begin{tabular}{ccc}
\hline $\begin{array}{c}\text { Roll Error } \\
{[\mathrm{deg}]}\end{array}$ & $\begin{array}{c}\text { Pitch Error } \\
{[\mathrm{deg}]}\end{array}$ & $\begin{array}{c}\text { Yaw Error } \\
{[\mathrm{deg}]}\end{array}$ \\
\hline $0.747^{\circ}$ & $0.854^{\circ}$ & $0.582^{\circ}$ \\
\hline
\end{tabular}

\section{B. Data Collection and Preprocessing Program}

A computer program records the data stream from the Central Board over Bluetooth [15]. The software implements Quaternion-based Extended Kalman Filter (EKF) to estimate the WB-4R orientation. The data acquisition for the different sensors, data preprocessing, EKF, data saving, and display processes are carried out on separate threads. The program further allows calibration of the IMU components, namely the gyroscope, accelerometer and magnetometer[15].

\section{Experimental Setup}

The subjects wore the sensors and sat upright on a chair in a room with an ambient temperature of $25^{\circ} \mathrm{C}$. A direct relationship has been observed between hyoid movement and bolus volume up to $10 \mathrm{ml}$ [3]. Hence, $10 \mathrm{ml}$ of water at room temperature was administered for each recording using a 25 $\mathrm{ml}$ graduated needleless syringe. For each subject, five repetitions of three trials were made at different head-neck angles. For the three trials, while sitting upright, the subjects were instructed to: 1 . Keep the head at a neutral rest position facing forward, 2. Rotate the head upwards to a chin up position, 3. Rotate the head downwards to a chin tuck position.

Before proceeding further, subjects were given a demonstration of the IMU and EMG sensor use. The real-time data of their actions, head movement and swallowing, was shown to them on the Software GUI. The EMG electrode placement was verified by having the subjects perform dry 
swallows and also swallows with a small uncontrolled water bolus.

Before starting each recording, the bolus was administered and the subject was instructed to keep it in the mouth. Each swallowing recording was 10 second long. The subject was asked to swallow by the experimenter at the 5-second mark of the recording.

\section{RESULTS}

The post processing was performed using a MATLAB script that loaded the raw IMU, Optotrak and EMG data files automatically and arranged them into a structure.

\section{A. IMU}

From EKF implementation, the orientation quaternion was calculated and then converted into a rotation matrix. Following that, by simple transformation, the relative rotation matrix between the IMUs mounted on the neck and head was acquired. This was then converted into Tait-Bryan angles of $\mathrm{XYZ}$ convention, representing the roll, pitch and yaw angles. The angles were passed through a 128-point moving average filter implemented with zero-phase forward and reverse digital IIR filtering.

Additionally, some non-angular parameters were also considered such as the acceleration norm and the jerk norm.

\section{B. Optotrak}

For the Optotrak, though four markers were used for redundancy, only three were enough to specify a reference frame as in Fig. 1. Since the markers can be obstructed by a number of environmental factors and experimental conditions, for every repetition and for every sensor, the marker with the most missing data was ignored and only the remaining three markers were used. Rotation matrices were calculated for the Optotrak frames and the relative angles between the two clusters of markers were acquired. The angles were filtered with the same scheme as the IMU angles.

\section{C. $E M G$}

In the present study, we examined the EMG activity in the suprahyoid muscles during swallowing. The EMG signal was rectified and smoothed with a moving average filter with a window size of 100 . The onset of the sEMG signal is used to recognize the swallowing event. For the sake of brevity, we have shown selected frames to demonstrate the relevant data.

\section{Data Analysis}

The estimated angles from the IMU and Optotrak were plotted and compared against each other, along with the EMG signal timeline for each of the three positions; Neutral, Chin down and Chin up. A sample of the IMU and Optotrak is shown with superimposed pitch angles from the three different positions in Fig. 3. In this figure, the green plots show the IMU pitch angles, while the magenta plots represent the corresponding Optotrak pitch angles.

We also compared the Roll, Pitch and Yaw angles for the IMU and Optotrak and computed the RMS Error for all the repetitions. These errors were averaged over repetitions across the subjects (Table II).

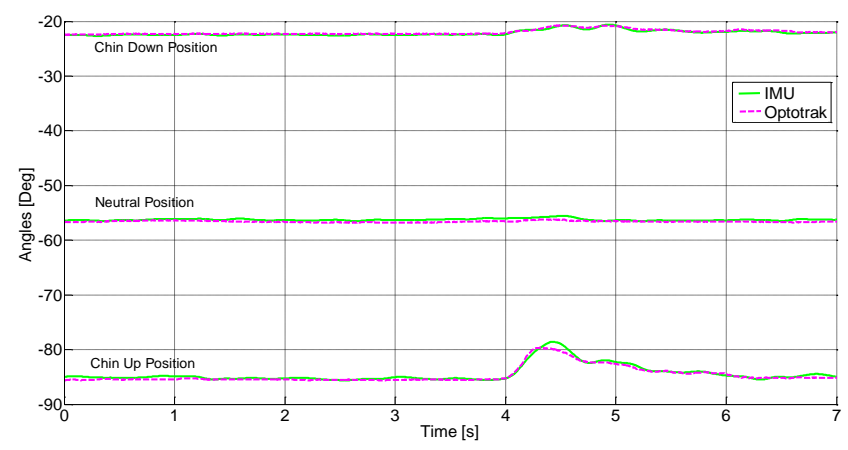

Fig. 3 Example of recording IMU and Optotrak Head-neck relative pitch angles for the three different head positions.

Additionally, the Acceleration Norm and Jerk Norm were also considered (Fig. 4) during deglutition and displayed alongside EMG signal to see any variability due to deglutition.
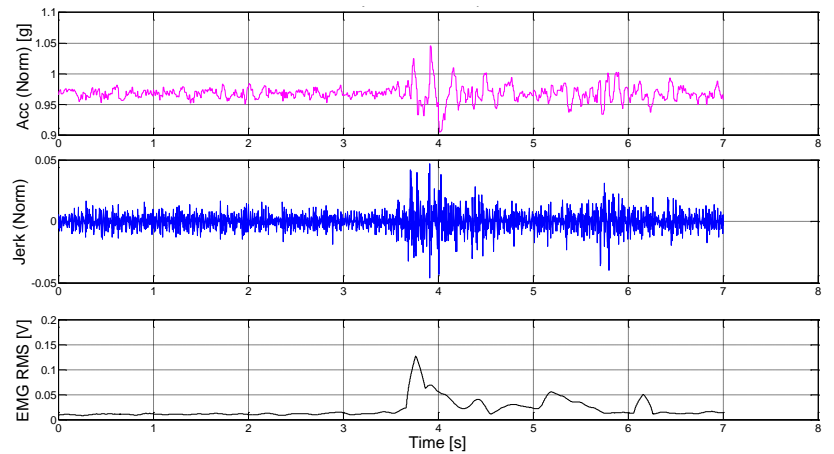

Fig. 4. Example of Acceleration norm(Top) and Jerk Norm(Center) during swallowing, alongside EMG RMS (Bottom)

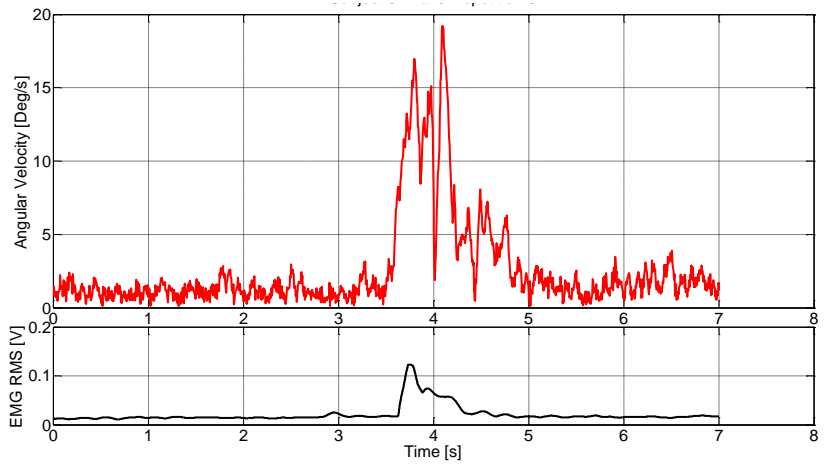

Fig. 5. Example of recording Gyroscope data during deglutition a. Angular velocity from Head mounted IMU Gyroscope, b. RMS EMG signal

Finally, we also compared the gyroscope sensor norm values, corrected for offset and gain, and filtered with an 8-point moving average, at the deglutition event (Fig. 5).

\section{DISCUSSION}

We can determine the head-neck posture in terms of Euler angles using this system. For the three different head positions, where the most important term is the pitch angle, it can be determined with high accuracy $\left(0.26^{\circ}\right.$ Averaged RMSE) as shown by the preliminary data. Change in pitch angle, corresponding to the head movement, can also be observed during the deglutition event. The roll and yaw angles exhibit errors of a few degrees $\left(1.63^{\circ}\right.$ and $1.24^{\circ}$ Averaged RMSE Respectively), This is because there is an influence of the magnetometer in determining these angles, 
Table II AVERAGED RMS ERROR OF WB-4R COMPARED WITH OPTOTRAK

\begin{tabular}{|c|c|c|c|c|c|c|c|c|c|c|c|c|}
\hline Positions & & Neutral & Position & & & Chin & Down & & & Chi & Up & \\
\hline Angle & Roll[deg] & Pitch[deg] & Yaw [deg] & $\mathrm{Excl}^{1}$ & Roll[deg] & Pitch[deg] & Yaw [deg] & Excl $^{1}$ & Roll[deg] & Pitch[deg] & Yaw [deg] & Excl $^{1}$ \\
\hline Subject 1 & 2.332 & 0.307 & 1.868 & $0 / 5$ & - & - & - & $5 / 5$ & 2.898 & 0.324 & 2.585 & $1 / 5$ \\
\hline Subject 2 & 1.161 & 0.211 & 0.683 & $0 / 5$ & 0.531 & 0.203 & 0.329 & $0 / 5$ & 3.445 & 0.323 & 2.972 & $1 / 5$ \\
\hline Subject 3 & 1.255 & 0.231 & 0.931 & $0 / 5$ & - & - & - & $5 / 5$ & 1.698 & 0.21 & 1.014 & $2 / 5$ \\
\hline Subject 4 & 1.305 & 0.2 & 0.705 & $0 / 5$ & 0.486 & 0.281 & 0.52 & $0 / 5$ & 1.795 & 0.321 & 1.353 & $0 / 5$ \\
\hline
\end{tabular}

which can vary owing to environmental factors and stray electromagnetic fields.

Since there was a single Optotrak sensor available, in some cases, more than one marker from a frame could get occluded. In such cases, it would not be possible to determine all the angles. Hence we had to exclude some data and average over the remaining while computing RMSE (Table II).

Using this system it is possible to measure parameters during the deglutition phase, such as the acceleration and the jerk norms. However, in many recordings, the acceleration norm is not distinct enough to be recognized as a swallowing event. So, the jerk norm can be a considerable parameter.

We found that there was some slight movement during deglutition that could be picked up by the gyroscope. As muscles in the neck region responsible for swallowing are also used for other physiological movements, the EMG for muscles in this region can be disturbed by other movements. For swallowing event detection using EMG, time domain features such as maximum and peaks may be useful to analyze [9]. However, owing to environmental and subject physiological factors, it may be difficult to obtain a good EMG recording in many cases. In such cases, we find that the swallowing event can be detected using the gyroscope instead.

\section{CONCLUSION}

We presented a novel approach to using an IMU and EMG based wearable sensor system for monitoring deglutition activities. As swallowing movement is known to arise from the contribution of different muscles and ligaments, to effectively understand the swallowing mechanism there is a need to get quantitative data about the different parts involved. This novel application, in deglutition study, of IMU sensors for head and movement and posture detection along with the accompanying EMG signals aims to provide exactly that.

We have shown in this work, the potential use of IMU and EMG sensors for measuring the head posture and muscle activity during deglutition. We have used the proposed sensor system for individual swallow events measurement. However, in the future, we intend to apply this method for monitoring for longer periods. We believe that this procedure can contribute towards better understanding and research of swallowing in humans.

Further works will adopt additional sensors of different types and sensor fusion methods for better identification of the swallowing events. Moreover, we plan to test the system efficacy further by using many additional test subjects.

\section{ACKNOWLEDGMENT}

This research has been conducted at the Waseda University Center for Advanced Biomedical Sciences, TWIns. The authors would like to express their thanks to the Italian Ministry of Foreign Affairs, General Directorate for Cultural
Promotion and Cooperation, for its support to RoboCasa. The authors would also like to express their gratitude to Life Performance Research, Okino Industries LTD, Japan ROBOTECH LTD, SolidWorks Corp, Dyden, and Kyoto Kagaku Co. Ltd. for their support to the research.

\section{REFERENCES}

[1] F. M. S. Mcconnel, "Analysis of pressure generation and bolus transit during pharyngeal swallowing," The Laryngoscope, vol. 98, no. 1, pp. 71-78, 1988.

[2] D. N. Ku, P.-P. Ma, F. M. S. McConnel, and D. Cerenko, "A kinematic study of the oropharyngeal swallowing of a liquid," Ann. Biomed. Eng., vol. 18 , no. 6, pp. 655-669, Nov. 1990

[3] W. Dodds, K. Man, I. Cook, P. Kahrilas, E. Stewart, and M. Kern, "Influence of bolus volume on swallow-induced hyoid movement in normal subjects," Am. J. Roentgenol., vol. 150, no. 6, pp. 1307-1309, Jun. 1988.

[4] H. Taniguchi, T. Tsukada, S. Ootaki, Y. Yamada, and M. Inoue, "Correspondence between food consistency and suprahyoid muscle activity, tongue pressure, and bolus transit times during the oropharyngeal phase of swallowing," J. Appl. Physiol., vol. 105, no. 3, pp. 791-799, Sep. 2008.

[5] M. Pehlivan, N. Yüceyar, C. Ertekin, G. Çelebi, M. Ertaş, T. Kalayci, and I. Aydoğdu, "An electronic device measuring the frequency of spontaneous swallowing: Digital Phagometer," Dysphagia, vol. 11, no. 4, pp. 259-264, Sep. 1996.

[6] C. Ertekin, A. Keskin, N. Kiylioglu, Y. Kirazli, A. Y. On, S. Tarlaci, and I. Aydoğdu, "The effect of head and neck positions on oropharyngeal swallowing: A clinical and electrophysiologic study," Arch. Phys. Med. Rehabil., vol. 82, no. 9, pp. 1255-1260, Sep. 2001.

[7] K. Sato and T. Nakashima, "Human adult deglutition during sleep," Ann. Otol. Rhinol. Laryngol., vol. 115, no. 5, pp. 334-339, May 2006.

[8] H. Firmin, S. Reilly, and A. Fourcin, "Non-invasive monitoring of reflexive swallowing," Speech Hear. Lang. Work Prog., vol. 10, pp. 171-84, 1997.

[9] O. Amft and G. Tröster, "Recognition of dietary activity events using on-body sensors," Artif. Intell. Med., vol. 42, no. 2, pp. 121-136, Feb. 2008.

[10] Z. Lin, M. Zecca, S. Sessa, L. Bartolomeo, H. Ishii, and A. Takanishi, "Development of the wireless ultra-miniaturized inertial measurement unit WB-4: Preliminary performance evaluation," in 2011 Annual International Conference of the IEEE Engineering in Medicine and Biology Society,EMBC, 2011, pp. $6927-6930$.

[11] Platzer, Werner. Taschenatlas Anatomie, Band 1: Bewegungsapparat. Georg Thieme Verlag, 2013.

[12] T. Pozzo, A. Berthoz, and L. Lefort, "Head stabilization during various locomotor tasks in humans," Exp. Brain Res., vol. 82, no. 1, pp. 97-106, Aug. 1990.

[13] U. Imtiaz, L. Bartolomeo, Z. Lin, S. Sessa, H. Ishii, K. Saito, M. Zecca, and A. Takanishi, "Design of a wireless miniature low cost EMG sensor using gold plated dry electrodes for biomechanics research," in 2013 IEEE International Conference on Mechatronics and Automation (ICMA), 2013, pp. 957-962.

[14] H. Takatsuji, H. M. Zakir, R. M. Mostafeezur, I. Saito, Y. Yamada, K. Yamamura, and J. Kitagawa, "Induction of the Swallowing Reflex by Electrical Stimulation of the Posterior Oropharyngeal Region in Awake Humans," Dysphagia, vol. 27, no. 4, pp. 473-480, Dec. 2012.

[15] W. Kong, K. Saito, Z. Lin, M. Zecca, S. Sessa, U. Imtiaz, S. Cosentino, L. Bartolomeo, H. Ishii, T. Ikai, and A. Takanishi, "Development of a real-time IMU-based motion capture system," in 2013 IEEE International Conference on Robotics and Biomimetics (ROBIO), 2013. 